\title{
THE REHYDRATION OF CHILDREN
}

\author{
By K. R. Llewellin, M.B., B.S., M.R.C.P. \\ Senior Medical Registrar, Westminster Children's Hospital
}

Rehydration literally implies the restoration of water alone. In simple depletion this is indeed all that is necessary, but in most cases of ' dehydration ' in childhood there also occur excessive losses of substances other than water. In this article the factors regulating the loss and restoration of these substances will be considered with a brief reference to the associated problems of acidbase balance and calorie intake. Much of the account will centre on the problem of rehydration in gastro-enteritis. Finally, a short description will be given of some of the technical procedures to be used.

\section{Water Depletion}

The fluid requirements of early infancy can be calculated with sufficient accuracy on the basis of $2 \frac{1}{2} \mathrm{oz}$. per lb. of expected body weight per day up to a weight of $\mathrm{I}_{5} \mathrm{lb}$. The expected weight of the infant can be computed by reckoning an ounce per day gain from the tenth to ninetieth day, and then a gain of $\mathrm{I} \mathrm{lb}$. per month till the end of the first year. The infant between 15 and $19 \mathrm{lb}$. requires $2 \frac{1}{4} \mathrm{oz}$. per lb. daily.

In later childhood the fluid requirements are proportionately less, being $2 \mathrm{oz}$. per lb. at I year; I.5 oz. per lb. at 4 years; and I oz. per lb. at ro years. At these ages ' expected weight' has to be calculated from actual weight, allowing for dehydration. In addition to maintenance fluid requirements a dehydrated child needs a 'restoration quota.' Where the amount of recent weight loss is known this additional need can be readily estimated, otherwise it has to be based on clinical assessment. The facies and skin turgor are the most useful signs, the state of the mouth and fontanelle having to be interpreted with caution; hyperpnoea may dry the former and meningitis fill the latter.

In infants dehydration suggests a fluid loss equivalent to 4 per cent. of the original body weight; severe dehydration 6 per cent.; and very severe dehydration 8 per cent. The restoration quota should be administered within four hours:

Example: A severely dehydrated, three-month- old baby weighs $9 \mathrm{lb}$. Its birth weight was $7 \mathrm{lb}^{-}$ but its weight immediately preceding the illness is unknown. As the dehydration is severe the predehydration weight can be estimated as

$$
\frac{9 \times 100}{92}=\text { 10 lb. approx. }
$$

The restoration quota is therefore $16 \mathrm{oz}$., or 450 ccs. The infant's expected gain from birth $=$ 80 oz. (i.e. 90-10), giving an expected weight of $12 \mathrm{lb}$. Maintenance intake is therefore $12 \times 2 \frac{1}{2} \mathrm{oz}$. $=30 \mathrm{oz}$., or $830 \mathrm{ccs}$. This baby should therefore be given for four hours fluid at the rate of $4 \mathrm{oz}$. or I Io ccs. per hour, i.e. about 30 drops per minute if by drip. Thereafter maintenance fluid is given at $1 \frac{1}{4} \mathrm{oz}$. $(35 \mathrm{ccs})$. per hour (approx. Io drops pers minute). Examples of pure water depletion can be found in cases where the child is taking an in-? adequate feed, not associated with diarrhœa or vomiting.

The water required in a particular case can be estimated from the above data. In regard to the administration of the restoration quota remember:

(a) That the quantity is related to both the size of the baby and the severity of dehydration-if in the example above, dehydration had been moderate $8 \mathrm{oz}$. would have sufficed in the first four hours.

(b) Urine output should be increasing rapidly towards the end of that period.

In most cases oral feeds of glucose water will prove adequate, the adoption of a $7 \times 3$ hourly or Io $x 2$ hourly schedule being often an advantage. Tube feeding may be necessary or, in cases where the oral-route is inadequate, part or all of the required fluid may be given by the subcutaneous or intravenous routes. For the intravenous route 5 per cent. glucose is the solution of choice but, unless absorption is expedited by hyaluronidase, sugar solutions should not be administered subcutaneously.

Sodium, Chlorine and Potassium Depletion and the Acid-Base Balance

Sodium is the chief cation and chlorine the 
chief anion of the extra-cellular fluid. Potassium is the chief cation of the intracellular fluid.

Upon the proper quantitative balance of these substances depends the normal osmotic equilibrium that exists between the intra-cellular, interstitial and intravascular fluids. Disproportionate loss of one or the other of them will, in addition, disturb the acid-base balance. Loss of potassium has a profound effect on muscle excitability. Dangerous electrolyte loss results from excessive sweating or excessive loss of alimentary canal secretion.

(I) If loss of gastric secretion, as in the vomiting of pyloric stenosis, is the dominant feature the principal loss is $\mathrm{HCl}$. The tendency to alkalosis thereby produced is compensated, with variable success by two mechanisms:

(a) By diminished respiratory loss of $\mathrm{CO}_{2}$ (hypopnoea).

(b) By increased renal excretion of base (decreased formation and secretion of $\mathrm{NH}_{4}$, excretion of $\mathrm{Na}_{2} \mathrm{HPO}_{4}$ rather than $\mathrm{NaH}_{2} \mathrm{PO}_{4}$, and excretion of base 'covered' by urinary carbonic acid).

The net result is a loss of sodium chloride from the interstial and intravascular fluids, the consequent fall in osmotic pressure determining the secondary dehydration and oligaemia of salt depletion.

There is an increase in the alkali reserve. The normal alkali reserve values in infancy, 45-55 vols. $\%(20-25 \mathrm{~m} \mathrm{Eq} / \mathrm{L})$, are somewhat lower than the adult range. Treatment in almost all cases resolves itself into supplying all the necessary $\mathrm{Cl}$ in the form of sodium chloride, and allowing the normal 'balancing action' of the kidney to restore the acid-base equilibrium. It is to be remembered that in persistent vomiting not due, to obstruction at the pylorus the loss of alkaline duodenal juice will produce a tendency to acidosis. In deciding how much and what kind of a salt solution to administer it is well to consider the salt and fluid requirements separately.

If we take as an example the baby considered under water depletion (but assuming that the dehydration is secondary to vomiting with production, therefore, of associated salt depletion):

Baby aged 3 months with expected weight of I $2 \mathrm{lb}$., an actual weight of $9 \mathrm{lb}$.; severely dehydrated so that its calculated recent weight is approximately ro $\mathrm{lb}$.

Expected maintenance fluid intake $=$ approx. $30 \mathrm{oz}$.

Expected maintenance salt intake (see below) $=\mathrm{r} .0 \mathrm{gm}$.

If the fluid intake is given as, say, 4.3 per cent. glucose $+1 / 5 \mathrm{~N}$. Saline the salt requirements will be well covered-so far as maintenance needs are concerned. If this child received its maintenance fluid intake in the form of normal saline its daily salt intake would be approximately 7.0 grammes. Oversaturation with salt is still an all too frequent and fatal blunder.

As the mechanism producing the dehydration in this case is vomiting there will be almost certainly an associated salt deficit. The correcting fluid should therefore be, say, 5 per cent. glucose $+\frac{1}{2}$ N. Saline. This should be administered only during the phase of rehydration, i.e. $45^{\circ}$ ccs. in four hours: approx. 2 grammes of salt.

Will this be sufficient to correct the salt deficit? If, in fact, this has been achieved the urinary chloride concentration will rise despite the increased urinary output associated with absorption of the restoration quota. This urinary chloride concentration can be estimated by means of the Fantus test. To ten drops of urine add one drop of 20 per cent. potassium dichromate (as indicator) then, drop by drop, 2.9 per cent. silver nitrate solution. The number of drops of the latter solution required to produce a yellow $\rightarrow$ red-brown colour change is supposed to be a measure of the number of grammes of chloride (as $\mathrm{NaCl}$ ) per litre of urine.

Apart from the fact that the test measurese chloride and not sodium excretion (and in chronico diarrhoea this is a severe limitation of its value experience soon shows that the claims that have been made for it, as a quantitatively accurate test are, in fact, over-optimistic. At least such is the case in infants. It remains a valuable though merely qualitative indication that salt reserves are depleted and are not rising in the body. The presence of one or more 'grammes per litre' in fairly dilute urine is an indication for the substitution of $\mathrm{I} / 5 \mathrm{~N}$. Saline +4.3 per cent. glucose for $\frac{1}{2}$ N. Saline +5 per cent. glucose. Once the phase of severe depletion is passed salt balance will be maintained by the following daily intakes :

Neonate, 0.25-0.5 gm.; I-3 months, 0.5-1.0 gm.; 3-12 months, $1-2 \mathrm{gm}$.; then a progressive rise through childhood to the adult IO-I5 gm. In all cases it is advisable at the outset to obtain blood from either the internal or external jugular veins or from the femoral vein, for estimation of the serum chlorides and alkali reserve. If the history of vomiting is a prolonged one serum proteins should be estimated as well. If the baby's condition contra-indicates venepuncture, sufficient blood may be obtained from a heel stab for the above estimations.

Recently it has been pointed out (Danowski, et al., 195 I) that in severe vomiting potassium losses may be considerable, and that serum potassium levels may not indicate such depletion. They quote a case of pyloric stenosis which, on ad- 
mission, had a normal serum potassium, but which developed a hypokalaemia on the administration of potassium-free fluids. Balance data suggested that during his illness the baby had lost about onefifth of his estimated total body potassium. In such cases the administration of a potassium solution similar to Darrow's but without the sodium lactate would be indicated, of course after the re-establishment of good renal output.

\section{Diarrhoea}

In diarrhoea there is a loss of $\mathrm{Na}$ and, in lesser degree, $\mathrm{Cl}$ from the extracellular fluid. There is also a shift of $\mathbf{K}$ from the intracellular to extracellular fluid, with partial loss to the alimentary canal.

The absolute loss of electrolyte from the tissues and the consequent fall in osmotic pressure brings about secondary dehydration and oligaemia in a manner similar to vomiting, but the predominant loss of base brings about a concomitant acidosis. The respiratory and renal mechanisms described in alkalosis of vomiting are brought into play, but in the reverse direction:-

(a) Increased respiratory loss of $\mathrm{CO}_{2}$ (hyperpnoea).

(b) Decreased renal excretion of base (increased formation of $\mathrm{NH}_{4}$ and excretion of $\mathrm{NaH}_{2} \mathrm{PO}_{4}$ rather than $\mathrm{Na}_{2} \mathrm{HPO}_{4}$ ).

The loss of $\mathrm{K}$ is particularly characterized by circulatory failure and hypotonicity. If $\mathrm{NaCl}$ is used as the correcting substance in a case of severe and prolonged diarrhoea the preceding disproportionate loss of $\mathrm{Na}$ will falsify the results of the Fantus test in that a high urinary excretion of chloride may be accompanied by low blood sodium levels. Clearly, in such a case, serum sodium levels should be determined. Further, the loss of $\mathrm{K}$ which will inevitably occur in such a case will not be made good if $\mathrm{NaCl}$ alone is used. Particularly as a result of the work of Darrow, potassium solutions have come into use in such cases. Darrow's solution consists of :-

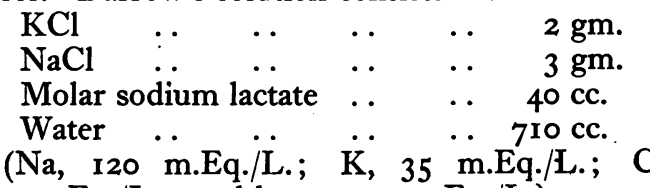
105 m.Eq./L.; and lactate, $52 \mathrm{~m}$. Eq./L.)

The normal serum potassium, I9-21 $\mathrm{mgm}$. per cent. ( $5 \mathrm{~m} . \mathrm{Eq}$./L.) may fall to $15 \mathrm{mgm}$. per cent. $(4 \mathrm{~m} . \mathrm{Eq} . / \mathrm{L}$.) or less in severe diarrhoea. If over correction occurs danger of heart block exists at around levels of $35-40 \mathrm{mgm}$. per cent. (8-10 m.Eq./L.). It is therefore essential :-

(a) Never to administer parenteral potassium solutions until a good urinary output has been established. (b) So to regulate the intake of potassium as to prevent excessive blood levels being reached.

This implies that the 'restoration quota' should be administered as $\frac{1}{2} \mathrm{~N}$. saline +5 per cent. glucose. If urinary excretion is well established by this measure, then the administration of a potassium solution can be commenced. Darrow recommends the subcutaneous infusion of his potassium fluid at the rate of $36 \mathrm{cc}$. per lb. in the first 24 hours, followed by 22 cc. per lb. on subsequent days, so long as parenteral therapy is necessary. Total fluid requirements, calculated in the usual way, are met by the additional administration of 5 per cent. glucose intravenously. As oral feeds are introduced the first fluid used is made up of one part of potassium solution to two parts of 5 per cent. glucose. With the introduction of milk feeds, I gm. of $\mathrm{KCl}$ is added to each day's intake until the calorie value of the feed has reached $35 \mathrm{cal}$. per lb. body-weight.

Many workers prefer to administer the parenteral potassium solution as part of the intravenous. infusion. A convenient plan would be to give fluid in four three-hourly periods, in each of which period the potassium solution is administered in a dosage of 9 cc. per lb., alternating with threehourly periods during which 5 per cent. glucose is administered in sufficient amount to cover total requirements. This on the first day; on subsequent days the dose of Darrow's solution in each period would be reduced to $5.5 \mathrm{cc}$. per $\mathrm{lb}$. A further alternative is to administer equivalent daily quantities of $\mathrm{KCl}$ as a continuous drip of lower concentration. It is highly desirable, but not essential, that potassium administration should be controlled by frequent estimations of the serum potassium. Methods in general use today are too slow to be of much value in guiding therapy, but techniques based on flame photometry promise greater rapidity, and the same basic technique can be utilized for the estimation of serum and urinary sodium levels.

The acidosis that is so frequently a feature of diarrhoeic states will, in most patients, respond to the above therapy. In patients where it is of great severity $\left(\mathrm{CO}_{2}\right.$ combining power of less than $9 \mathrm{~m}$. Eq./L. or 20 vol. per cent.), one-sixth molar sodium lactate should be used initially during administration of the restoration quota. Given a dosage of $27 \mathrm{cc}$. per lb., this solution will raise the $\mathrm{CO}_{2}$ combining power by approximately $30 \mathrm{vol}$. per cent.

\section{Calorie Requirements and the Introduction of \\ Oral Feeds}

The normal calorie requirements in infancy can be reckoned on a basis of 50 calories per $\mathrm{lb}$. of expected weight during the first four months and 
45 calories per $\mathrm{lb}$. from the fifth to the twelfth month. In older children the normal range at each age level becomes respectively greater, but approximate figures may be given as follows: at three years 1,400 calories per day, at five years 1,600 calories per day; and at ten years 2,300 calories per day.

Five per cent. glucose solution has a calorie value of 6 calories per oz.; clearly from the point of view of adequate calorie intake the early establishment of oral feeding is important; present-day tendencies are undoubtedly in that direction. Oral feeds are safer than parenteral infusions. Anorexia, vomiting and abdominal distension are the principal contra-indications to oral feeding. The passage of large, frequent and loose stools is another, but it would be wrong to await for normality in stool formation before commencing feeds-one might wait indefinitely. Just how rapidly feeds can be increased depends on the history of the case. An acute onset, with rapid regression of symptoms on the institution of treatment, indicates a rapid introduction and increase in the size of feeds. A child, even if quite severely dehydrated prior to the establishment of parenteral therapy, may be fit to receive half its fluid requirements orally on the second day, and all on the third day. On the second day 4.3 per cent. glucose $+\mathrm{I} / 5 \mathrm{~N}$. saline should be the oral fluid, or, if diarrhoea has been a feature of the case, the I : 2 potassium-glucose mixture described under potassium deficit.

On the third day $\frac{1}{4}$ strength half-cream National Dried Milk (i.e. I measure to $4 \mathrm{oz}$. water) can be used. This has a calorie value of ro calories per lb. expected weight. Subsequent feeds are: on the fourth day half-strength, half-cream National Dried Milk (20 calories per lb.): on the fifth day three-quarters strength, half-cream National Dried Milk (30 calories per lb.). In diarrhoeic cases I gm. $\mathrm{KCl}$ should be added to the day's feed until this stage. On the sixth day full-strength, halfcream National Dried Milk (40 calories per lb.) is given. This last formula should be used for one week. If progress is satisfactory sugar can then be added, in the proportion of one teaspoonful to every $5 \mathrm{oz}$. of feed. This 47 calories per lb. mixture should be used for a fortnight, when a gradual return can be made to the diet normal for the child's age: until this stage is reached vitamins should be added to the feeds, several proprietary preparations being quite convenient and satisfactory.

When a history of symptoms is more protracted and their disappearance slower on parenteral therapy the introduction of oral feeds has to be correspondingly more cautious. It may be eight to ten days before the 40 calories per $\mathrm{lb}$. weight formula is reached and the parenteral administrations of protein, and possibly blood, assumes great importance. These will be considered in the following sections. In these cases, and especially if aureomycin, chloromycetin, or terramycin have been used additional Vitamin B complex should be given.

\section{Protein Administration}

The type of case needing early protein intake has been indicated above. Estimation of serum proteins will be of value in indicating the presence of severe depletion; oedema may be due to hypoproteinaemia. Total serum proteins are normally lower in the neonate than in the adult. In the premature baby the normal serum protein level is 4-5 gm. per cent., consequently oedema levels are readily reached. The protein requirements of early infancy can be reckoned on a basis of $1.8 \mathrm{gm}$. per lb. body-weight; at three years $1.6 \mathrm{gm}$. per lb.; in later childhood $1.2 \mathrm{gm}$. per lb. In infants and small children virtually all this protein should be of animal origin. If the child's condition precludes the oral route then use must be made of parenteral infusions of plasma or of casein hydrelysate. Disadvantages attach to each of these alternatives. Pooled plasma conveys the virus of hepatitis with sufficient frequency to make the possibility a worrying one-in debilitated patients the disease can be very serious. Irradiation does not completely obviate this risk; no filtration technique has yet been evolved. Casein hydrolysate would appear to provide a perfect alternative; physically easier to transfuse than plasma, its use is not attended by any risk of hepatitis. However, a tendency to cause venous thrombosis, and the relative ease with which amino-acid 'spillover' into the urine can occur, are serious objections. My preference is for plasma; when administered I part to 3 parts of 5 per cent. glucose the solution contains I gm. of protein in every $2 \frac{1}{2}$ oz. and, in $\mathrm{NaCl}$ content, is equivalent to $1 / 6$ normal saline.

If salt depletion is suspected the diluting fluid should be 5 per cent. glucose $+\frac{1}{2} \mathrm{~N}$. saline, used only so long as urinary evidence of salt depletion is forthcoming. In diarrhoeic cases the addition of $0.75 \mathrm{gm}$. of $\mathrm{KCl}$ to every $560 \mathrm{cc}$. $(20 \mathrm{oz}$.) of the glucose-plasma solution provides a potassium intake comparable to the first day on the ordinary Darrow schedule. The addition of $0.45 \mathrm{gm}$. of $\mathrm{KCl}$ to every $560 \mathrm{cc}$. of solution should suffice for each subsequent day that the patient receives intravenous therapy. The usual check on serum potassium levels should be observed, i.e. daily estimation while the intravenous potassium intake is at a high level, where facilities exist for so doing. The gradual introduction of oral feeds permits the 
use of half-cream National Dried Milk (I gm. of protein per measure of milk powder). This can be reinforced by:

Casilan (protein 26 gm./oz.) and Prosol (protein I9 gm./oz.), practically tasteless whole protein preparations, or Hepavite (protein 15 gm./oz.), hydrolysate with malted flavour, and Oral Casydrol (protein 14 gm./oz.), hydrolysate with cheesy flavour.

In considering which preparation to use the great variation in price should be remembered.

\section{Indications for Blood Transfusion}

Many children who suffer dehydration as the result of an infective process develop a severe anaemia; many ' $D$. and V.' infants have suffered from a preceding nutritional anaemia. The exhibition of large doses of iron is often contraindicated in these children, and, even when vell tolerated, there is often a poor response. In assessing an infant in this respect it should be remembered that the normal haemoglobin level between the second and fifth months is of the order of 70 or 80 per cent.; however an infant may have a haemoglobin not far below these levels, yet benefit considerably from a small blood transfusion.

If a 'one-shot' technique exploiting a scalp vein is adopted then a dosage of ro cc. per lb. should be used. If a drip transfusion is used the following formula can be adopted:

$$
\frac{\mathrm{IOO}-\mathrm{H}}{\mathrm{I} 00} \times 40 \times \mathrm{W}=\text { volume of blood required }
$$

in cc., where $\mathrm{H}=$ haemoglobin in percentage Haldane, $\mathrm{W}=$ infant's weight in pounds.

With this technique the rate of administration should not exceed 12 drops per minute or $1 \frac{1}{2} \mathrm{oz}$. per hour. Signs of over-transfusion are: variation in pulse rate, especially marked slowing; dyspnoea; distension of neck veins; cyanosis; basal crepitations; enlargement of the liver and peripheral oedema. At the appearance of the first of these signs the drip should be slowed to six drops per minute and the baby carefully observed. If improvement is not immediate the drip should be stopped. The other aspects of blood transfusion differ in no way in children as compared with adults.

\section{Technical Procedures}

Technique of obtaining blood from heel prick. In the routine management of the dehydrated child careful assessment of the clinical features gives sufficient guidance for correct therapy. When a case presents with atypical features, or where response to treatment is unsatisfactory, determination of the serum chloride, serum sodium and

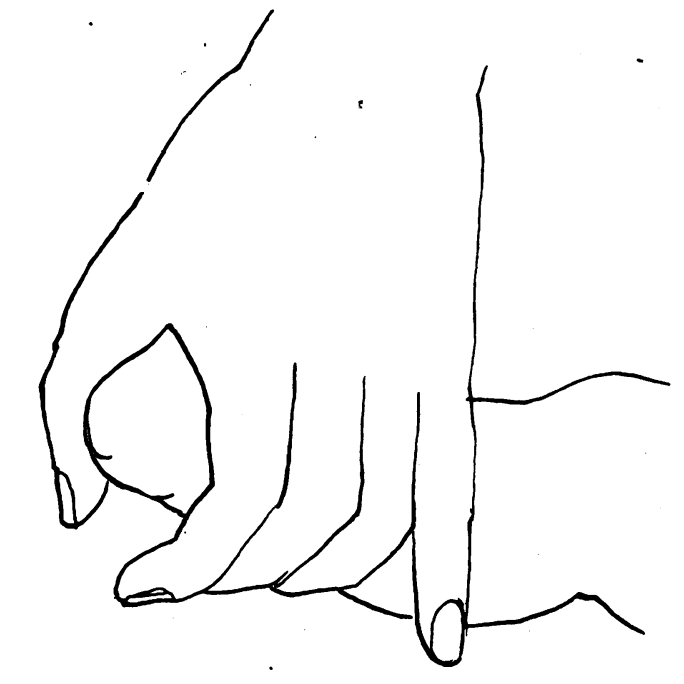

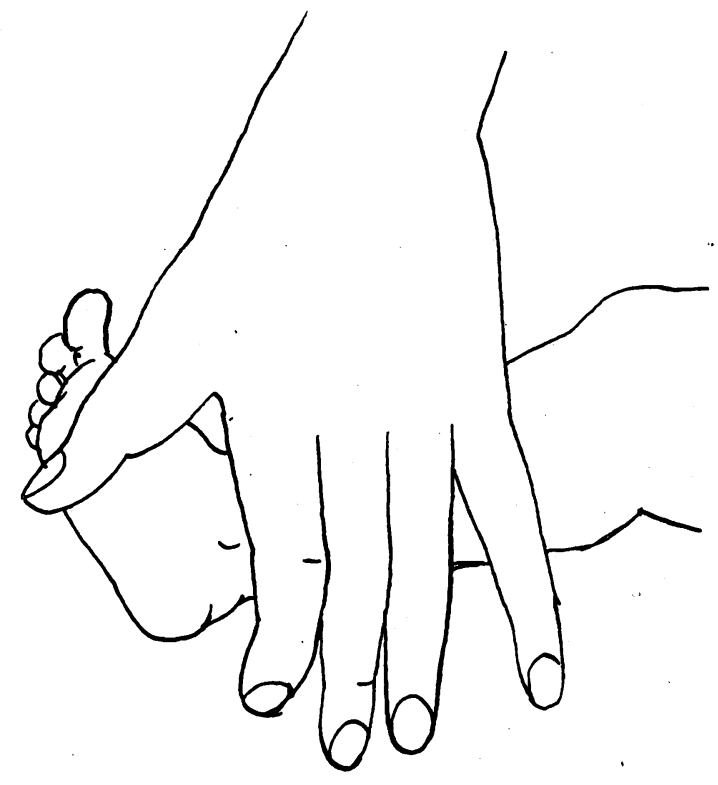

FIG. 1.-Shewing the expression technique for obtaining blood by heel puncture: note the full relaxation allowed between each period of pressure. 


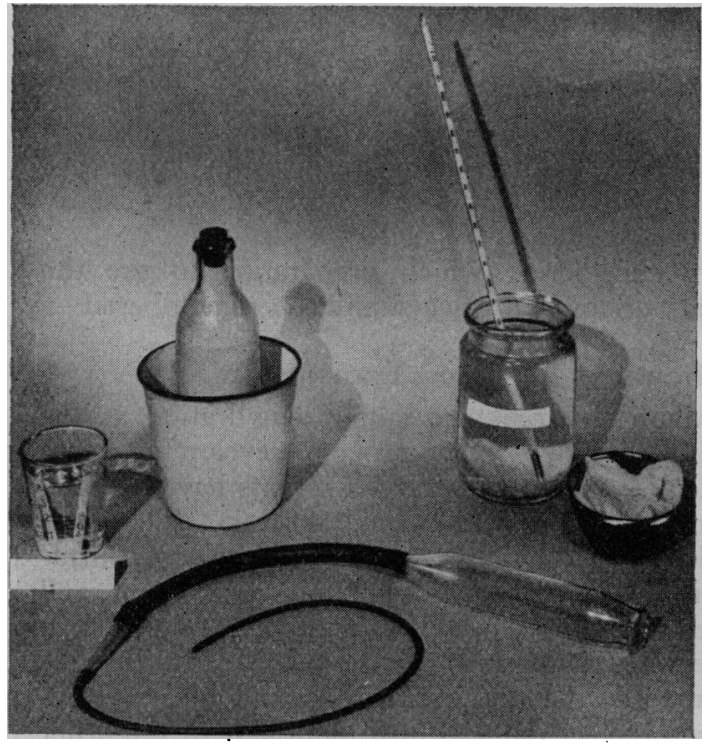

FIG. 3. -The essential apparatus for tube feeding.

Fig. 2.-The type of tube used for collecting blood after heel puncture.

serum potassium, and estimation of the alkali reserve and blood urea may be of great importance. Venous blood from the internal jugular or external jugular or femoral vein is the most satisfactory for these tests, but if, for any reason, difficulty is experienced in obtaining blood, a heel prick may be satisfactory. The baby's heel should be first warmed by immersion in warm water for two minutes. It should then be rapidly dried and a thin smear of sterile vaseline applied to the heel (this latter measure aids the collection of the blood as discrete drops). The heel should be firmly pricked with a Hagedorn's needle and the expression technique illustrated in Fig. I should be carried out. The importance of completely relaxing between each compression is shown by the illustration. The blood should be collected into one or other of the tubes shown in Fig. 2.

Tube feeding. In cases where inability to swallow is the principal bar to the maintenance of hydration, tube feeding may be of the greatest value. It is not without danger; it should not be ordered by a medical officer who has no practical experience of the procedure, nor, should a nursing staff, similarly ignorant, be expected to carry it out. It undoubtedly has its greatest sphere of usefulness in the immature neonate, but some workers have used it extensively, often by drip-feed technique, in older children. Dangers of the method lie in misdirection of the tube, over-rapid disten-

sion of the stomach, regurgitation and inhalation of feed, and injury to mucosa. The essentrik apparatus is indicated in Fig. 3. Nasal or pêoral intubation should be decided on the followingo basis:

(a) The nasal route is absolutely contra-⿳亠口冋 indicated in the neonatal group. In such cases ao

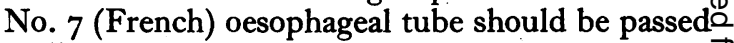
orally.

(b) In older children the nasal route is often 3 preferable as the tube is better tolerated in this position. It should not be used for repeated in-? tubation. A No. 5 (French) catheter is suitableo in most cases.

(c) In older children, where the nasal route for any reason is contra-indicated, a No. 8 oeso-i phageal tube can be used.

The apparatus, as illustrated, should be assembled and washed through with sterile water. With the child in a sitting position the catheter or $\frac{}{8}$ oesophageal tube should be passed rapidly. For nasal intubation a trace of lubricant should bes applied to the tube; one nares may prove easier than the other. Force is unnecessary and injurious. For an intra-gastric feed the tube should $\omega$ be passed an inch further than the distance from? the mouth to the ensiform cartilage. An escape of gas will indicate that the tube is in the stomach. A somewhat shorter length should be passed for an oesophageal feed. Coughing or change of colour on the part of the baby is an indication for 


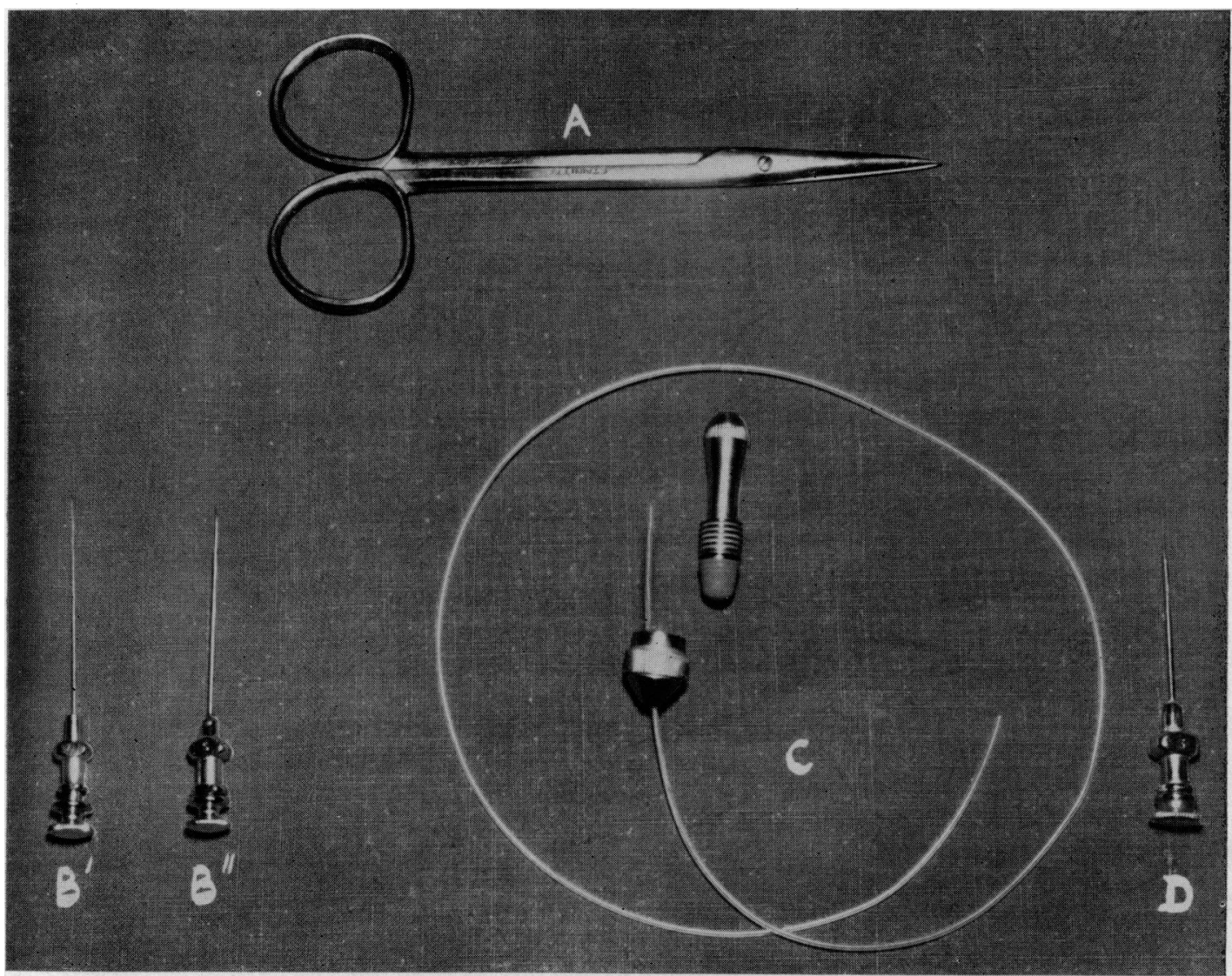

FIG. 4.-Instruments for intravenous infusions in infants and young children. $\mathbf{B}^{\prime}, \mathbf{B}^{\prime \prime}$, Bateman's needle: C, polythene tubing and adzptor: $\mathrm{D}$, modified lumbar puncture needle.

the immediate withdrawal of the tube. As a further precaution a small quantity of sterile water should be passed before the feed.

Oesophageal feeding prevents the over-rapid distension of the stomach and is especially indicated in the more feeble or immature neonate. The last portion of the feed is ' followed down' by a small quantity of sterile water; the tube is then rapidly withdrawn.

\section{Subcutaneous Infusions}

This route can be relied upon to contribute only a part of the child's total daily fluid requirements. Using hyaluronidase total needs may be met, but not with certainty. Subcutaneous infusions are useful in two particular roles :-

(a) As a means of satisfying potassium requirements with safety-as in the Darrow technique.

(b) It may supplement oral feeds, either in the mild case of dehydration or subsequent to the use of the intravenous route in more severe cases.
The axillae, abdomen or thighs can be used. A Y-shaped connection with double needles is an advantage. If hyaluronidase is used I $\mathrm{mgm}$. should be injected into the drip tubing immediately proximal to the needle. In slowly absorbed subcutaneous infusions the use of glucose is attended by a grave risk of cellulitis.

\section{Intravenous Infusions}

The paediatrician has a choice of an astonishing variety of sites and techniques; however, the basic technique of which mastery is essential for anyone dealing with sick children is the cutdown intravenous drip using a small, rigid cannula or polythene tubing. The most reliable vein is the internal saphenous vein at the internal malleolus. As far down as the needle adaptor the infusion apparatus is the standard M.R.C. infusion set. The large needle or cannula is replaced either by a Bateman needle or by an adapted fine lumbar puncture needle (Fig. 4 d) or by polythene tubing (Fig. 4). 


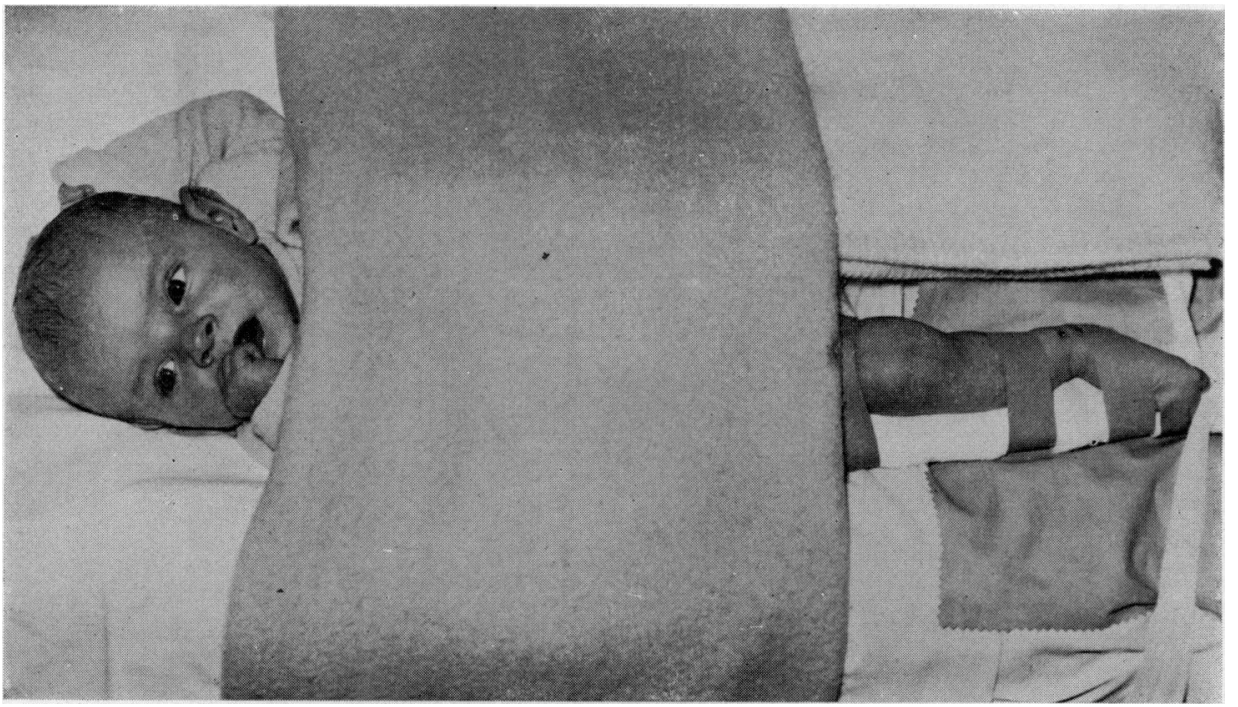

Fig. 5.-Shewing the method of splinting the leg for an infusion into the internal saphenous vein at the ankle

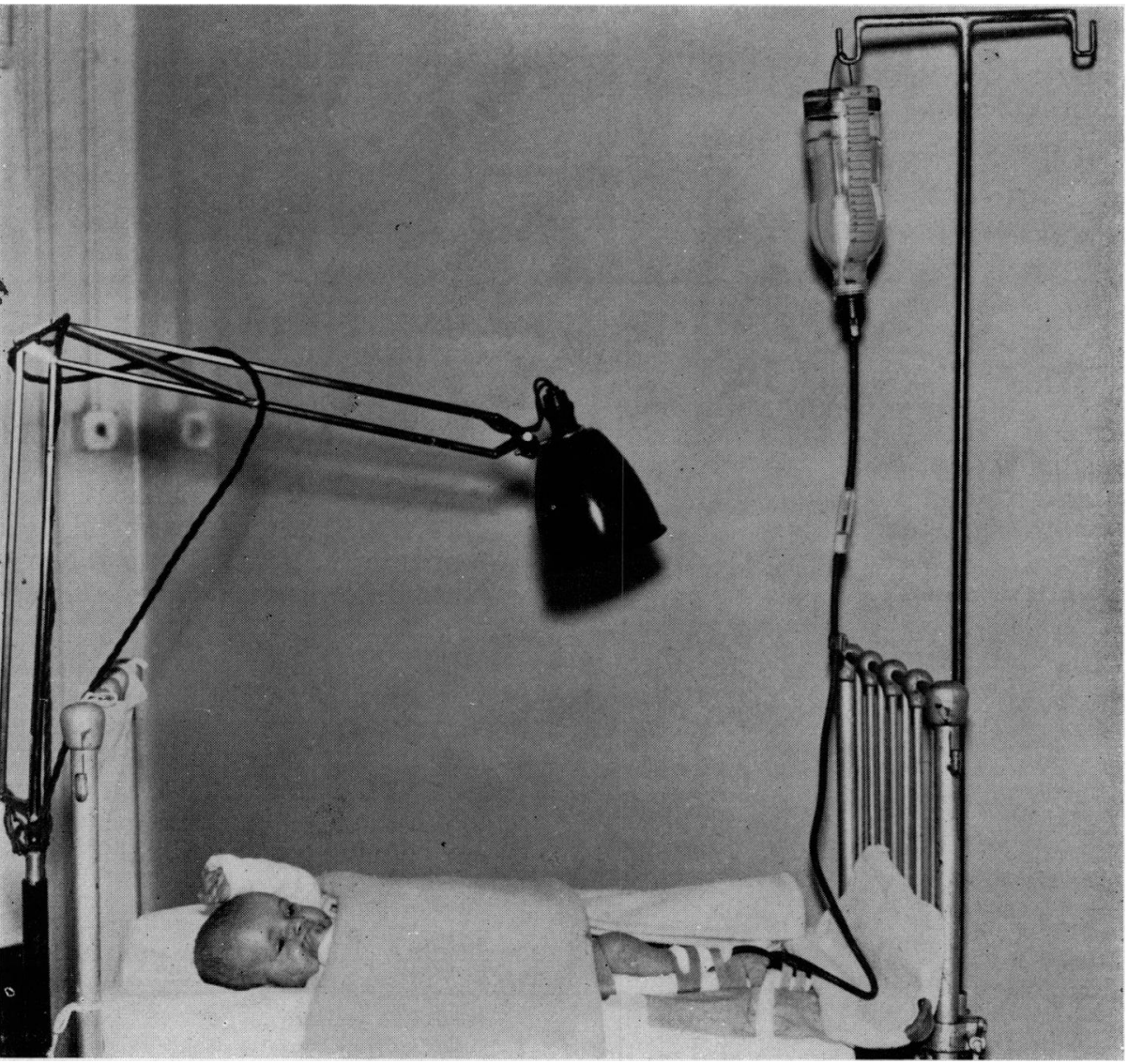

Fig. 6.-An intravenous drip assembled. Note (a) the splinting, (b) the use of an Anglepoise lamp, (c) the ounce graduations on the bottle. 
The Bateman ' needle' consists of an outer cannula $\left(B^{\prime \prime}\right)$ which is introduced into the vein, and an inner cannula $\left(B^{\prime}\right)$ which is attached to the drip set. Clearly such an arrangement facilitates the changing of defective or obstructed drip apparatus. It is a widespread practice nowadays to use only the outer Bateman, thus minimizing the degree of obstruction to the infusions of, especially, blood and plasma. A lumbar puncture needle of bore intermediate between the outer and inner Bateman cannulae, suitably shortened and with the point rounded off, is very useful. Because of the ease with which it adapts itself to the course of the vein, and because a sufficient length can be passed to make rigid splinting of the leg unnecessary, polythene tubing (sizes I or 2) has much to commend it. It is claimed that its use is attended by a higher incidence of thrombophlebitis, and that the tubing tends to 'fur up' rather easily. In my experience it has proved more convenient and less attended by undesirable sequelae than the rigid cannulae. The fact that the tip of the polythene tubing usually lies in the lumen of a bigger vein than would the tip of a rigid cannula, appears to make for easier running.

It is important to carry out the assembly of an infusion in an unhurried fashion. The less frequently the operation is performed in a unit the greater are the chances of leaks and misfits in the drip apparatus and defects in the cutting down set. It should not be necessary to 'assemble' the requisite instruments; full cutting-down sets should be available already sterilized. The splinting of the child's limb should be done by the doctor himself, with the ward sister's supervision, at least until he is familiar with the possible snags. Chloral sedation is often valuable; a restless baby of three months will need 2 gr.

It is important that the child should be kept adequately warm throughout the procedure-an 'Anglepoise' lamp (Fig. 6) serves usefully both to warm and light the leg. Elsewhere well-protected hot water bottles may be necessary. In blood transfusion, especially, overheating must be avoided. If oxygen therapy is deemed necessary the tent should not be discontinued for the cutdown; a re-arrangement of the tent should permit access to arm or leg. A well-padded splint which projects for some distance beyond the limb should be used. The thigh should be slightly abducted in full external rotation; the leg should be slightly flexed; the foot plantar-flexed and inverted. While the thigh and leg are held by the nurse the foot should be firmly strapped in the above position. The leg and thigh can be immobilized either by a well-padded bandage or preferably a series of encircling non-elastic adhesive tape strips. If the latter are used it is important that the tape overlap should rest on underlying tape and not on skin; if, as rehydration proceeds, it becomes necessary to slacken these bands, undue pulling upon the skin is thus avoided. It should be possible to remove the baby from the cot for the purpose of feeding, etc., without unduly disturbing the drip.

Local anaesthesia can be attained by infiltration of the surrounding area with I per cent. procaine. The drip apparatus should then be assembled, connected with the infusion bottle and checked to see that it is running normally. When the bottle has been suspended, a piece of adhesive tape, calibrated so as to measure the contents of the bottle in ounces, is fixed to the side of the bottle. The initial fluid level should be noted.

The patency of the cannula should be assured; if polythene tubing is to be used it should be removed from cetavlon and rinsed with sterile water carefully cleansing both lumen and exterior. It should next be threaded into and fixed in the adaptor shown (Fig. 4c). Next make certain that the catgut ligatures and aneurysm needle are handy and that a syringe loaded with normal saline is available. Lastly, before making the incision, see that the light is in a good position and that one is in a comfortable working position.

A transverse incision shown is made through the full thickness of the skin. From then on pro ceed by blunt dissection, using an artery forceps with a light action; a stiff forceps makes for early finger fatigue and clumsiness. Usually, but not invariably, the internal saphenous vein is fairly constant in regard to surface marking; its depth from the surface is very variable. The presence of a thread-like parallel tributary vein lying superficial to the true vein may prove very confusing to the inexperienced. If the vein cannot be found after a patient search, the incision should be enlarged posteriorly. When the vein is located it should be defined over a distance of perhaps $\frac{1}{2}$ in.; overenthusiasm in cleaning the vein may precipitate troublesome spasm and should be avoided. With the aid of the aneurysm needle, two strands of catgut are passed behind the vein. The distal ligature is tied occluding the vein; the free ends of the ligature are held in artery forceps. This provides a useful ' handle' by which the vein can be controlled. The proximal ligature is half-tied and again anchored by an artery forceps. The vein is now put on tension by gentle traction on the distal ligature, and a transverse snick made in it with fine scissors. The success of this step, and of the whole procedure, depends on the sharpness and precise functioning of this instrument (Fig. 4a), which should be used for no other purpose. The vein should be hemi-sectioned at the first attempt; nibbling precipitates spasm and so lacerates the vessel wall that cannulization becomes almost impossible. 
Retrograde blood flow is rarely of sufficient degree to be an inconvenience; the Bateman cannula or polythene tubing is now gently threaded into the vein. If a rigid cannula is used it should be allowed to 'follow the vein' even if it means an awkward angle; polythene of course does so anyway, and about 4 in. of this should be passed. Sometimes, but not invariably, blood will flow from the end of the cannula. The infusion tubing should now be connected and the screw clip opened. If the infusion does not run :-

(a) Check the apparatus for the presence of an air lock.

(b) Make sure that the limb is not unduly compressed.

(c) Try gentle variation of the angle of the cannula.

(d) 'Prove' the vein by gentle injection of normal saline through the cannula by syringe.

(e) Warm the leg.

Syringing through a vein is only justifiable at this stage; while it may be attempted with success in a drip that has stopped after several hours' running, under such circumstances the procedure is too dangerous. Fixation of the cannula and of the tubing immediately beyond the limb, is of great importance. Narrow strips of adhesive tape serve very well. The wound itself should be dusted with flavazole or penicillin-sulphathiazole powder and covered with sterile gauze and a bandage.

The rate of drip is often rather variable in the early stages of a transfusion and needs constant checking. The number of ounces infused in eight hours is approximately indicated by the number of drops per minute; but the calibrated tape is an essential check on the quantity given. During the phase of restoration the rate may be relatively high - of the order of 20 to 30 drops per minute. Maintenance rates are in the order of 10 to 15 drops per minute.

During this early stage oral fluids will probably consist only of drachm doses; as the oral contribution increases the parenteral quota is proportionately reduced. The keeping of a comprehensive fluid intake and ouput chart is essential.

Observation of this fluid balance is a serious responsibility which rests upon the nursing staff; equally important is their immediate reporting of any untoward change in the baby's condition or any suggestion of blockage in the drip.

If the drip slows down after running for several hours the possibilities causal of slow starting should be considered, except that syringing through the vein is contra-indicated at this stage. The leg should be re-examined for developing oedema and the dressing loosened if necessary.

Any development of an inflammatory reaction around the drip wound or further up the leg is an imperative indication for stopping the infusion. In a small and debilitated baby a suppurative thrombo-phlebitis or cellulitis may proceed veryo quietly until the calf is a sack of pus; if the infecting organism is Ps. pyocyanea the outlook is especially grave. The safeguard is meticulous aseptic technique and care, but not meddlesome observation of the drip; the value of a chemotherapeutic ' umbrella' is controversial, but some experienced operators are convinced of its value.

I am grateful for the help given me by $\mathrm{Mr}$. Levi, Dr. Beautyman and Sister Hammond, and to Dr. Hansell who provided the photographs, and my wife for the line drawings.

\section{REFERENCES}

Technical Procedures

GARROD, BATTEN and THURSFIELD (1947), 'Diseases of Children,' Vol. I, Chap. VIII.

Water, Elestrolyte, and Acid-Base Balance (v.s.) Chap. VI

MARRIOTT, H. L. (1950), 'Water and Salt Depletior..'

GAMBIE, J. L. (1947). 'Chemical Anatomy, Physiology and Pathology of Extraceliular Fluid.'
Potassium Therapy

DARROW, D. C. (1949), Paediatrics, 4, 604-6r 9.

DARROW, D. C. (1948), Bull. N.Y. Med., 24, 147-165.

DANOWSKI, T. S. (1951), Acta Paediatrica, 40, 198-21 2.

Blood Transfusion

WHITBY and BRITTON (1950), 'Disorders of the Blood.'

Early Oral Feeding

CHUNG, A. W. (1948), f. Paediatrics, 32, I. 